\section{Evaluating the impact of a fungal-origin chitosan preparation on Brettanomyces bruxellensis in the context of wine aging}

\author{
Tiziana Nardi,' Paola Vagnoli,' \\ Andrea Minacci,, Sandrine Gautier, ${ }^{3}$ \\ Nathalie Sieczkowski ${ }^{4}$
}

${ }^{1}$ Lallemand Inc. Italia, Castel d'Azzano (VR); 'ISVEA Srl, Poggibonsi (SI), Italy;

${ }^{3}$ KitoZyme, Herstal, Belgium; ${ }^{4}$ Lallemand SAS, Blagnac, France

\section{Abstract}

Brettanomyces bruxellensis and the consequences of its development in wines are a continuous threat for wine quality. In this context, chitosan of fungal origin was introduced as a new tool to control B. bruxellensis in the context of winemaking. Recent studies have showed the impact of a fungal origin chitosan application on wines contaminated with $B$. bruxellensis, leading to the elimination of $B$. bruxellensis cells. In these studies, the chitosan preparation was added, the wine racked off after 10 days and the efficiency of the treatment was evaluated in a short delay after the treatment. This study focused on the evaluation of the impact of different addition protocols of an enological chitosan preparation on $B$. bruxellensis population evolution and volatile phenols content along the aging, up to 9 months. The results confirm the interest of fungal origin chitosan as a preventive tool to control B. bruxellensis in the context of wine aging.

\section{Introduction}

Brettanomyces bruxellensis and the consequences of its development in wines are a continuous threat for wine quality. ${ }^{1}$ This undesirable yeast is able to develop during aging under difficult conditions. Development of this microorganism usually results in the production of off-flavors, such as ethylphenols and vinylphenols; ${ }^{2,3}$ in fact, the sensory properties of these molecules (mousiness, animal, horsy, barnyard, smoky, spicy, burnt plastic, or medicinal) are often described as the Brett character. ${ }^{4}$ It is also responsible for the production of other negative aromatic compounds such as isovaleric acid (known to be related to un unpleasant cheesy aroma) and tetrahydropyridines, responsible for mousy taint. $^{5}$ Nowadays at an international scale, volumes concerned by this defect become significant, and $B$. bruxellensis is considered as the major microbial cause for wine spoilage worldwide, causing significant economic losses. ${ }^{6} B$. bruxellensis is well adapted to winemaking conditions since it is low $\mathrm{pH}$ and ethanol tolerant, facultatively anaerobic, ${ }^{7}$ and it can assimilate carbon sources alternative to hexoses. ${ }^{8}$ Several strategies are useful to control $B$. bruxellensis and its development in musts and wines, although they are not always sufficient. ${ }^{1,9}$ In this context, chitosan of fungal origin has been introduced as a new potential tool to control $B$. bruxellensis in winemaking. ${ }^{10}$ Chitosan is a linear polysaccharide composed of two repeating units [D-glucosamine units (GlcN) and $\mathrm{N}$-acetyl-D-glucosamine (GLcNAc) units] randomly distributed along the polymer chain and linked by $\beta(1-4)$-bonds. Recent studies have showed the impact of chitosan application $^{9}$ on wines contaminated with $B$. bruxellensis, leading to the elimination of $B$. bruxellensis cells, even at high levels of populations up to $10^{5}-10^{6} \mathrm{CFU} / \mathrm{mL} .^{11}$ In these studies, the chitosan preparation was added, the wine racked off after 10 days and the efficiency of the treatment was evaluated in a short delay after the treatment. Due to the necessity to control wine microbiological stability during the period of aging in barrels, our research focuses on the application of an enological chitosan preparation in order to prevent wine from $B$. bruxellensis contamination along the aging period at both experimental winery- and winery-scale.

This study aims at evaluating the impact of different addition protocols of an enological chitosan preparation on $B$. bruxellensis population evolution and volatile phenols content along the aging, up to 9 months. Moreover, since previous studies have reported that the management of an efficient malolactic fermentation (MLF) can help to preserve the quality of wine by hampering the development of Brettanomyces yeast, ${ }^{12-14}$ the application of chitosan treatments in this work has been carried out both on wines that underwent rapid MLF (by yeast-bacteria co-inoculation) and on wines that underwent slow MLF (carried out by spontaneous microflora).

\section{Materials and Methods Chitosan of fungal origin}

The chitosan preparation used (No Brett Inside $^{\mathrm{TM}}$ ) is a powder with particles : whose diameter is lower that $50 \mu \mathrm{m}$, extracted from Aspergillus niger, produced by KitoZyme (Herstal, Belgium). Four $\mathrm{g} / \mathrm{hL}$ were added in the treated wines.

\section{Microorganism strains and culture conditions}

The strain of Oenococcus oeni used for wine
Correspondence: Tiziana Nardi, Lallemand Inc Italia, via Rossini 14B, 37060 Castel d'Azzano (VR), Italy.

Tel: +39.045.512555 - Fax: +39.045.519419.

E-mail: tnardi@lallemand.com

Key words: Brettanomyces bruxellensis, chitosan, wine aging.

Aknowledgements: this work was carried out thanks to the collaboration of Castello di Fonterutoli-Marchesi Mazzei winery, Castellina in Chianti (SI), Italy. Authors specially thank Dr. Luca Biffi, chief winemaker, who took care of the trials.

Presented at the $3^{\text {rd }}$ International Conference on Wine Active Compounds (WAC) - March $26^{\text {th }}-28^{\text {th }}$ 2014, Beaune, France.

Contributions: AM, performed the pilot-scale experimentations and the analytic follow-up of pilot- and winery-scale experimentations; SG, provided the fungal origin chitosan formulation TN, PV and NS, coordinated the experimentations (from experimental set-up to results interpretation).

Received for publication: 16 July 2014.

Revision received: 26 September 2014.

Accepted for publication: 29 September 2014.

This work is licensed under a Creative Commons Attribution 3.0 License (by-nc 3.0).

(C) Copyright T. Nardi et al., 2014

Licensee PAGEPress, Italy

Wine Studies 2014; 3:4574

doi:10.4081/ws.2014.4574

MF in this work was Lalvin VP41 ${ }^{\circledR}$ (Lallemand, Montreal, Canada). The bacteria were rehydrated from lyophilized form in distilled water for $20 \mathrm{~min}$, then added to the must or wine.

The strain of Brettanomyces bruxellensis used in this study was originally isolated from red wine and designed as DB 137 in the internal collection by ISVEA [Poggibonsi (SI) Italy]. The yeast was routinely grown in liquid yeast extract peptone dextrose medium $(10 \mathrm{~g} / \mathrm{L}$ yeast extract, $20 \mathrm{~g} / \mathrm{L}$ peptone, and $20 \mathrm{~g} / \mathrm{L}$ glucose, $\mathrm{pH} 4.8$ ). At the end of the exponential phase of growth, the yeast was inoculated into the wine mass at $1 \times 10^{3}$ colony forming units $(\mathrm{CFU}) / \mathrm{mL}$.

\section{Experimental design}

Several experimentations were run during 2 years in Tuscany, Italy (2011 and 2012). Experiments were set up at experimental-winery scale (25 L tanks) and winery-scale (225 L barrels) on Sangiovese and Merlot wines and followed along the aging (6 months at experimental winery, 9 months at winery). At winery scale, the impact of MLF was also taken into account, by comparing wines that underwent 
co-inoculated MLF and spontaneous MLF.

For each matrix (Sangiovese and Merlot, 2011), wine was dispatched in 3 different 25L stainless-steel tanks, different treatments were applied: control (untreated); chitosan 4 g/hL, no rack-off, no batonnage (stirring); chitosan $4 \mathrm{~g} / \mathrm{hL}$, no rack-off, batonnage once a week. After the treatments, each tank was inoculated with $B$. bruxellensis cells (preadapted to wine) at the rate of $10^{3} \mathrm{CFU} / \mathrm{mL}$.

At the end of alcoholic fermentation 2011, wines Merlot and Sangiovese were separated into 2 lots each. One lot was inoculated with selected lactic acid bacteria (Oenococcus oeni) and the other went through spontaneous MF. At the end of MLF, for each lot, wine was put into 3 separated barrels for 9 months aging, with different chitosan treatments : control (untreated); chitosan $4 \mathrm{~g} / \mathrm{hL}$, no rack-off, no batonnage; chitosan $4 \mathrm{~g} / \mathrm{hL}$, no rack-off, batonnage once a week. The same trial was conducted on a 2012 Sangiovese after MLF completed by co-inoculated lactic acid bacteria, on a larger number of barrels (4 per condition).

\section{Follow-up of the experiments}

Classical chemical parameters were analysed at the set-up of the trials and at the end of the aging period. The analytical methods used were those recommended by the International Organisation of Vine and Wine (OIV). Sugars were analysed by alkylamineresin HPLC (OIV-MA-AS311-03), ${ }^{15}$ alcohol by volume by densimetry using hydrostatic balance (OIV-MA-AS312-01A), ${ }^{16} \mathrm{pH}$ by potentiometry (OIV-MA-AS313-15) and sulfur dioxide (free and total) by iodometric titration (OIVMA-AS323-04B). ${ }^{17}$

Quantification of B. bruxellensis cells were performed at the set-up of the trials and every month during the aging period, by plating on selective medium after proper dilutions (modified DBDM-agar ${ }^{18}$ containing cycloheximide $50 \mathrm{mg} / \mathrm{L},{ }^{9}$ incubation for 10 days at $26^{\circ} \mathrm{C}$ ). Quantification of volatile phenols was performed at the set-up of the trials and at the end of the aging period by HPLC on an RP18 column with a fluorimetric detector. ${ }^{19}$

\section{Results and Discussion}

\section{Experimental-winery scale}

As a general remark on $B$. bruxellensis contamination, higher microbial populations were reached on Sangiovese compared to Merlot, although alcohol level and $\mathrm{pH}$ were similar and no residual sugars were present in the 2 wines (Merlot: alcohol 13.91\%, pH 3.41, residual sugars $0.90 \mathrm{~g} / \mathrm{L}$; Sangiovese: alcohol 13.78\%, pH 3.37 , residual sugars $0.86 \mathrm{~g} / \mathrm{L}$ ). In the experimental-winery experiment, where $B$. bruxel- lensis was inoculated on wines after the chitosan treatment, we observed a clear impact of chitosan on the evolution of $B$. bruxellensis population along the 6 months. The best control of $B$. bruxellensis contamination was achieved with batonnage on the Sangiovese wine, and with or without batonnage on the Merlot wine (Figure 1). It is important to mention that wines had been previously desulphit-
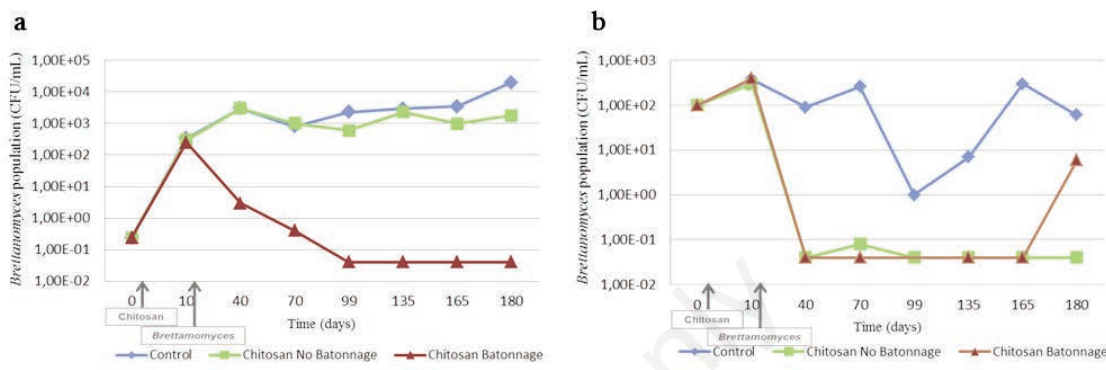

Figure 1. Results of the experiment conducted at experimental winery-scale in 2011: $B$. bruxellensis populations in Sangiovese (a) and Merlot (b) wines
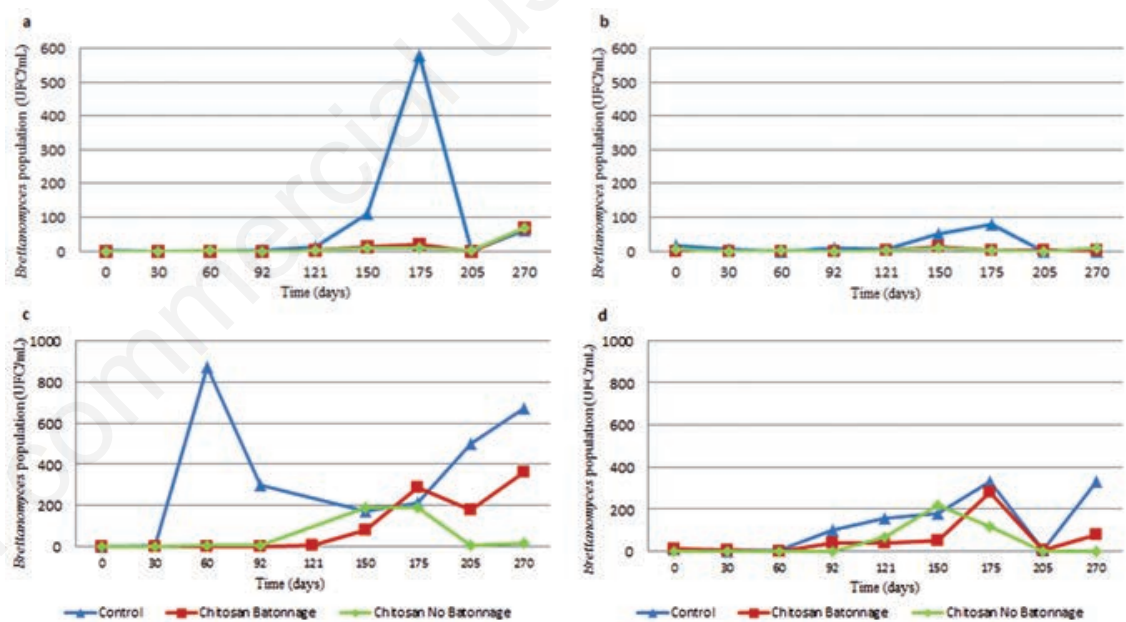

Figure 2. Results of the experiment conducted at winery-scale in 2011: B. bruxellensis populations in Merlot [spontaneous malolactic fermentation (a) and co-inoculation (b)] and Sangiovese [spontaneous malolactic fermentation (c) and co-inoculation (d)] wines.
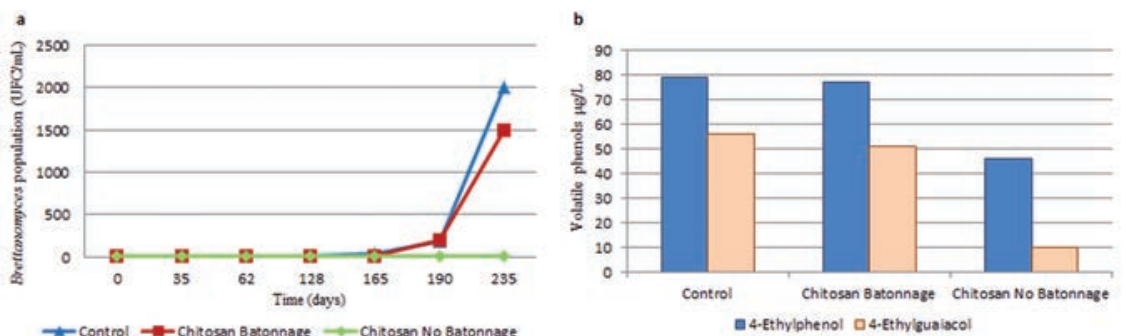

Figure 3. Results of the experiment conducted at winery-scale on the 2012 Sangiovese wine [Brettanomyces bruxellensis population follow-up (a) and final volatile phenols concentrations $(\mathbf{b})]$. 
spontaneous MLF. Chitosan addition, with or without batonnage, helped to prevent $B$. bruxellensis growth up to 6 months in this condition, especially in wine with spontaneous MLF (Figure 2a). On the 2011 Sangiovese wines (Figure 2c and d), where B. bruxellensis populations were slightly higher, it is interesting to notice the positive impact of bacteria co-inoculation on $B$. bruxellensis contamination, as previously described. ${ }^{11}$

Furthermore, B. bruxellensis development occurred earlier in wines non-treated with chitosan, confirming the impact of the compound. Again, it is important to mention that wines did not receive any $\mathrm{SO}_{2}$ addition during the 6 first months of aging.

Finally, wines were analysed for classical phisico-chemical parameters (data not shown) and tasted after the 9 months and no impact of the contact with chitosan was observed (volatile phenols remained under sensory threshold, data not shown).

\section{2-13 experiments}

Observations made on 2011 were confirmed on a 2012 Sangiovese wine, regarding the impact of chitosan on the long-term application to protect wines against $B$. bruxellensis. During aging, when kept in contact with wine, the addition of chitosan without batonnage led to a lower contamination of $B$. bruxellensis, (Figure $3 \mathrm{a}$ ) which is correlated with a lower volatile phenols concentration compared to the treatment with batonnage and to the control (Figure 3b).

\section{Conclusions}

The goal of this work was to evaluate a chitosan formulation as a tool to protect wines against $B$. bruxellensis along the time of aging. On the one hand, the pilot-scale experimentations simulated how a chitosan treatment could prevent from the growth of a following contamination with $B$. bruxellensis. The results show that the tested chitosan formulation may offer a preventive tool in order to protect the wine from Brettanomyces bruxellensis, when leaving the chitosan formulation in contact and resuspending it. Indeed, we observed that batonnage seemed to help to limit B. bruxellensis growth. On the other hand, the wineryscale experiment, where the contamination was natural (from wine and barrel), led to the conclusion that chitosan can be a tool to control $B$. bruxellensis in the context of aging in barrels along the period of aging. In that case, the treatment without stirring (batonnage) seems to lead to the highest inhibition, possibly due to the presence of a lower $B$. bruxellensis population. In any case, chitosan treatments allowed to keep Brettanomyces populations under control during the long-term contact, also limiting the rise of volatile phenols production by this yeast.

The whole data set of this work shows for the first time the impact of a long term contact (up to 9 months) of chitosan to prevent $B$. bruxellensis growth in in wine, as consistent results were observed in different grape varieties (Merlot and Sangiovese), different contamination conditions (artificial and natural) and on two subsequent vintages (2011 and 2012). Fungal origin chitosan preparation can be a useful tool for winemakers to $\operatorname{control} B$. bruxellensis in wines during aging, when coupled with a regular survey of microbial contaminations in wines. Also, these results confirm that a good management of MF during winemaking process (here achieved by yeast-bacteria co-inoculation) can be coupled as another preventing tool to avoid Brettanomyces growth. Further experiments would be needed to better clarify the impact of batonnage on the action of chitosan.

\section{References}

1. Suarez R, Suarez-Lepe JA, Morata A, Calderon F. The production of ethylphenols in wine by yeasts of the genera Brettanomyces and Dekkera: a review. Food Chem 2007;102:10-21.

2. Chatonnet P, Dubourdieu D, Boidron J, Pons M. The origin of ethylphenols in wines. J Sci Food Agr 1992;60:165-78.

3. Dias L, Dias S, Sancho T, et al. Identification of yeasts isolated from wine related environments and capable of producing 4-ethylphenol. Food Microbiol 2003;20:567-74.

4. Heresztyn T. Metabolism of volatile phenolic compounds from hydroxycinnamic acids by Brettanomyces yeast. Arch Microbiol 1986;146:96-8.

5. Romano A, Perello M C, de Revel G, Lonvaud-Funel A. Growth and volatile compound production by Brettanomyces/Dekkera bruxellensis in red wine. J Appl Microbiol 2008;104:157785.

6. Loureiro V, Malfeito-Ferreira M. Spoilage yeasts in the wine industry. Int $\mathrm{J}$ Food Microbiol 2003;86:23-50.
7. Woolfit M, Rozpedowska E, Piskur J, Wolfe $\mathrm{KH}$. Genome survey sequencing of the wine spoilage yeast Dekkera (Brettanomyces) bruxellensis. Eukaryot Cell 2007;6:721-33.

8. Conterno L, Joseph CML, Arvik TJ, et al. Genetic and physiological characterization of Brettanomyces bruxellensis strains isolated from wines. Am J Enol Viticult 2006;57:139-47.

9. Zuehlke JM, Petrova B, Edwards CG. Advances in the control of wine spoilage by Zygosaccharomyces and Dekkera/ Brettanomyces. Annu Rev Food Sci Technol 2013;4:57-78.

10. Gomez-Rivas L, Escudero-Abarca BI, Aguilar-Uscanga MG, et al. Selective antimicrobial action of chitosan against spoilage yeasts in mixed culture fermentations. J Ind Microbiol Biot 2004;31:16-22.

11. Blateyron L, Bornet A, Brandam C, et al. Le chitosane d'origine fongique: un nouvel outil de choix pour lutter contre Brettanomyces dans les vins. Revue des Oenologues 2012;143:27-8.

12. Gerbaux V, Briffox C, Dumont A, Krieger S. Influence of inoculation with malolactic bacteria on volatile phenols in wines. Am J Enol Viticult 2009;60:233-5.

13. López R, López-Alfaro I, Gutiérrez AR, et al. Malolactic fermentation of Tempranillo wine: contribution of the lactic acid bacteria inoculation to sensory quality and chemical composition. Int J Food Sci Technol 2011;46:2373-81.

14. Wedral D, Shewfelt R, Frank J. The challenge of Brettanomyces in wine. LWT-Food Sci Technol 2010;43:1474-9.

15. Tusseau D, Bouniol C. Determination des glucides dans les mouts, vins et champagnes par HPLC. Sci Aliment 1986;6:55977.

16. OIV. Compendium of international methods of wine and musts analysis. Paris, France: Office International de la Vigne et du Vin; 2013.

17. Ripper M. The determination of the total sulphur dioxide content of ciders. J Prakt Chem 1892;46:428.

18. Rodrigues N, Gonçalves G, Pereira-daSilva S, et al. Development and use of a new medium to detect yeast of the genera Dekkera/Brettanomyces ssp. J Appl Microbiol 2001;90:588-99.

19. Nicolini G, Larcher R, Bertoldi D, et al. Rapid quantification of 4-ethylphenol in wine using high-performance liquid chromatography with a fluorimetric detector. Vitis 2007;46:202-6. 